\title{
Povidone Iodine 'WET' technique: Adenotonsillectomy in COVID era
}

\author{
Nithya Venkataramani ${ }^{1}$, RAVI SACHIDANANDA ${ }^{2}$, and Nidhi Sachidananda ${ }^{3}$ \\ ${ }^{1}$ People tree hospital yeshwanthpur \\ ${ }^{2}$ People tree hospital, Bangalore \\ ${ }^{3}$ people tree hospitals bangalore
}

June 11, 2020

\begin{abstract}
Key Points Most of the ENT surgeries are aerosol generating procedures and pose a risk of infection to health care workers. Povidone-iodine irrigation has been used in surgery and has proven to reduce surgical site infection. Povidone iodine has good virucidal action even in low concentrations. The novel technique of wet tonsillectomy with povidone iodine flush would possibly reduce the viral load. This technique, in adjunct to other safety measures and personal protective equipment, could be a safe way to restart elective oral, ear and nasal procedures in asymptomatic patients during the pandemic.
\end{abstract}

\section{Povidone Iodine 'WET' technique: Adenotonsillectomy in COVID era}

Key Points

Most of the ENT surgeries are aerosol generating procedures and pose a risk of infection to health care workers.

Povidone-iodine irrigation has been used in surgery and has proven to reduce surgical site infection.

Povidone iodine has good virucidal action even in low concentrations.

The novel technique of wet tonsillectomy with povidone iodine flush would possibly reduce the viral load.

This technique, in adjunct to other safety measures and personal protective equipment, could be a safe way to restart elective oral, ear and nasal procedures in asymptomatic patients during the pandemic.

COVID 19 pandemic has significantly changed ENT Practice. Most elective ENT (Ear, Nose, Throat) surgeries possess a risk of infection, as well as potentially increasing complication (1). With the current pandemic slowly evolving and ever-increasing demand for elective services to restart, service delivery with minimal risk to healthcare workers is a challenge. ENT procedures like Adenotonsillectomy, Microdebrider FESS and Mastoidectomy and many more are aerosol generating procedures. Adenotonsillectomy is particularly challenging with high risk to the operating room personnel as the viral load is significantly high in the nasopharynx and oropharynx. Evidence from China shows us that the pandemic could last for at least six months(2) and modifications of techniques and adapting to the new norm is the key.

Some countries are now considering RTPCR as routine. The sensitivity of the test is questioned with false negative rate of up to $30 \%$ (3)It is therefore imperative to be cautious even in patients who are RTPCR negative. We propose a technique of 'WET' (wash every time) adenotonsillectomy with povidone iodine wash to minimise the viral load, if any, to healthcare workers while performing adenotonsillectomy surgery.

\section{Methods}




\section{Technique}

Once the patient is listed for Adenotonsillectomy, patients have to be screened in the OPD for history and for symptoms suggestive of COVID-19. This is done by a questionnaire as per institutional protocol. (Although we started ENT surgeries with COVID RTPCR screening initially, the current ICMR guidelines precludes routine screening). Patients needing adenotonsillectomy as per international guidelines are chosen for surgery. It is ensured that the patient does not have other comorbidities (Diabetes, Hypertension) especially thyroid illness. A preoperative surgical consent and COVID-19 consent are obtained from the patient (or parent). Patient is asked to gargle his/her mouth with $2 \%$ povidone iodine gargle three days before and just before shifting to the operating room. All the staff in operating room must wear the recommended PPE (personal protective equipment) which includes a cover all gown (2 layers), an N-95 mask or Respirators, gloves, and an eye cover. Intubation is done with standard anaesthetic precautions using an intubation Box. The patient is then positioned in Tonsillectomy position.

After application of a throat pack, a $2.5 \%$ povidone iodine is applied in the oral cavity and Nasal cavity for five minutes. Adenoidectomy is done using suction diathermy and tonsillectomy is done using bipolar dissection to minimize bleeding and blood flash. Throughout the surgery, $2.5 \%$ povidone iodine solution is flushed with a syringe into the operating field, (oral cavity and nasal cavity for Adenoidectomy), and is suctioned out intermittently(Figure 1). The surgery in carried out in the small pool of povidone iodine solution. Complete haemostasis is achieved and extubation is carried out with aerosol precautions. The patient is sent home the next day with antibiotics and Quarantine instructions.

\section{Discussion}

The ENT surgeon is at risk of COVID -19 infection during this pandemic both in OPD(outpatient) and the operation room(4) It is known that almost all ENT surgeries are aerosol generating, or surgeries are performed in areas which have close contact with nasopharynx or oropharynx. With an intent to reduce viral load in the patient, and to reduce the infection rate amongst the treating doctors, Povidone iodine has been suggested as a nasal spray and a mouth wash to health care workers to reduce the risk of infection(5).

In vitro studies on the efficacy of povidone iodine show that a concentration of $0.23 \%$ has good bactericidal and virucidal property $(6)$.

Povidone-iodine irrigation has been used in surgery and has proven to reduce surgical site infection. Although it is a simple technique, concerns were raised regarding absorption and impaired thyroid function, but until now, no serious concerns have been reported other than allergy(7).

Contamination of the povidone-iodine solution has been associated with infections with Pseudomonas, therefore, precautions should be taken to ensure its sterility before use(8),(9). In general, povidone-iodine irrigation can be used for wound irrigation in all patients except in patients with iodine sensitivity, burns, thyroid disease or renal disease .

We recommend a novel technique of wet tonsillectomy with povidone iodine flush, which would possibly reduce the viral load at the source before aerosol generation, thereby reducing risk to health care workers. Povidone iodine is easily available, economical, and is fairly safe in low concentrations, thus this could be a safe way to restart elective oral, ear and nasal procedures in asymptomatic patients during the pandemic. This is in adjunct to the recommended personal protective equipment and other safety measures in the operating room. 


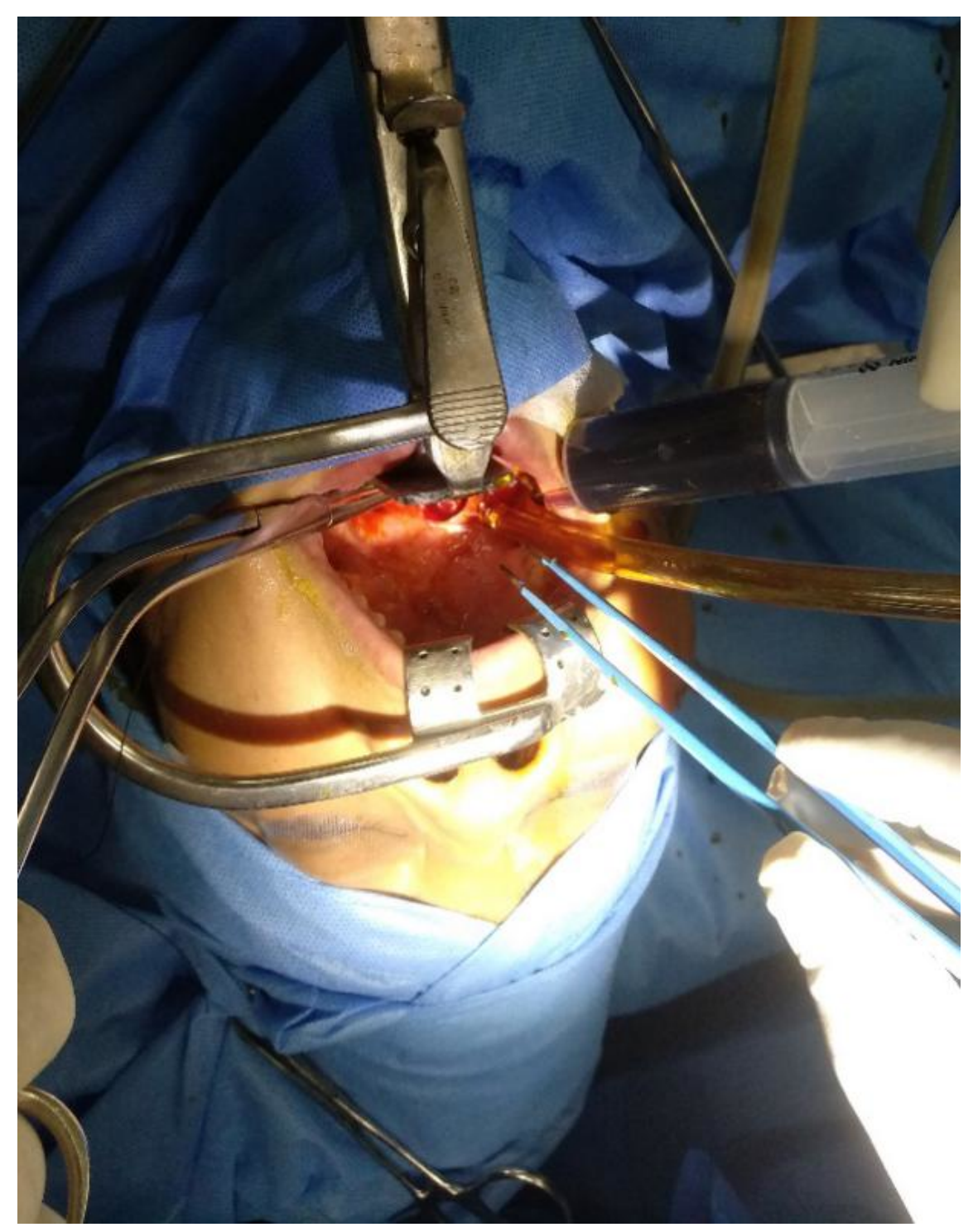

Figure 1: WET tonsillectomy with Povidone Iodine

\section{References}

1. Lei S, Jiang F, Su W, Chen C, Chen J, Mei W, et al. Clinical characteristics and outcomes of patients undergoing surgeries during the incubation period of COVID-19 infection. EClinicalMedicine. 2020 Apr;21:100331.

2. Reuters. China reports 51 new coronavirus cases, mostly in Wuhan. The Hindu [Internet]. 2020 May 25 [cited 2020 Jun 7]; Available from: https://www.thehindu.com/news/international/china-reports-over50-new-coronavirus-cases-mostly-in-wuhan/article31667737.ece

3. Woloshin S, Patel N, Kesselheim AS. False Negative Tests for SARS-CoV-2 Infection - Challenges and Implications. N Engl J Med. 2020 Jun 5;0(0):null.

4. Vukkadala N, Qian ZJ, Holsinger FC, Patel ZM, Rosenthal E. COVID-19 and the Otolaryngologist: Preliminary Evidence-Based Review. The Laryngoscope. 2020 Mar 26; 
5. Kirk-Bayley J, Sunkaraneni S, Challacombe S. The Use of Povidone Iodine Nasal Spray and Mouthwash During the Current COVID-19 Pandemic May Reduce Cross Infection and Protect Healthcare Workers [Internet]. Rochester, NY: Social Science Research Network; 2020 May [cited 2020 Jun 7]. Report No.: ID 3563092. Available from: https://papers.ssrn.com/abstract $=3563092$

6. Mady LJ, Kubik MW, Baddour K, Snyderman CH, Rowan NR. Consideration of povidone-iodine as a public health intervention for COVID-19: Utilization as "Personal Protective Equipment" for frontline providers exposed in high-risk head and neck and skull base oncology care. Oral Oncol. 2020 Jun;105:104724.

7. Chundamala J, Wright JG. The efficacy and risks of using povidone-iodine irrigation to prevent surgical site infection: an evidence-based review. Can J Surg. 2007 Dec;50(6):473-81.

8. Berkelman RL, Lewin S, Allen JR, Anderson RL, Budnick LD, Shapiro S, et al. Pseudobacteremia Attributed to Contamination of Povidone-Iodine with Pseudomonas cepacia. Ann Intern Med. 1981 Jul $1 ; 95(1): 32-6$.

9. Panlilio AL, Beck-Sague CM, Siegel JD, Anderson RL, Yetts SY, Clark NC, et al. Infections and Pseudoinfections Due to Povidone-Iodine Solution Contaminated with Pseudomonas cepacia. Clin Infect Dis. 1992 May 1;14(5):1078-83. 